\title{
Cortical Responses to Cochlear Implant Stimulation: Channel Interactions
}

\author{
Julie Arenberg Bierer and John C. Middlebrooks \\ Kresge Hearing Research Institute, Department of Otorhinolaryngology, University of Michigan, Ann Arbor, MI 48109-0506, \\ USA
}

Received: 12 November 2002; Accepted: 28 July 2003; Online publication: 20 October 2003

\section{ABSTRACT}

This study examined the interactions between electrical stimuli presented through two channels of a cochlear implant. Experiments were conducted in anesthetized guinea pigs. Multiunit spike activity recorded from the auditory cortex reflected the cumulative effects of electric field interactions in the cochlea as well as any neural interactions along the ascending auditory pathway. The cochlea was stimulated electrically through a 6-electrode intracochlear array. The stimulus on each channel was a single 80$\mu \mathrm{s} /$ phase biphasic pulse. Channel interactions were quantified as changes in the thresholds for elevation of cortical spike rates. Experimental parameters were interchannel temporal offset (0 to $\pm 2000 \mu \mathrm{s})$, interelectrode cochlear spacing (1.5 or $2.25 \mathrm{~mm}$ ), electrode configuration (monopolar, bipolar, or tripolar), and relative polarity between channels (same or inverted). In most conditions, presentation of a subthreshold pulse on one channel reduced the threshold for a pulse on a second channel. Threshold shifts were greatest for simultaneous pulses, but appreciable threshold reductions could persist for temporal offsets up to $640 \mu \mathrm{s}$. Channel interactions varied strongly with electrode configuration: threshold shifts increased in magnitude in the order tripolar, bipolar, monopolar. Channel interactions were greater for closer electrode spacing. The results have implications for design of speech processors for cochlear implants.

Current address (Julie Arenberg Bierer): Epstein Laboratory, Box 0526, University of California, San Francisco, CA 94143-0526, USA.

Correspondence to: John C. Middlebrooks, Ph.D. - Kresge Hearing Research Institute - University of Michigan - 1301 E. Ann Street Ann Arbor, MI 48109-0506. Telephone: (734)-763-7695; fax: (734)764-0014; email: jmidd@umich.edu
Keywords: cochlear implants, auditory cortex, guinea pig, channel interaction

\section{INTRODUCTION}

Present-day cochlear prostheses can provide a high level of speech recognition in patients that have severe-to-profound hearing loss. Nearly all prostheses that are in clinical use employ multiple stimulating channels, where a channel refers to a signal pathway involving one or more active and return electrodes; present-day implants comprise between 8 and 22 intracochlear electrodes, with or without an extracochlear reference electrode. Multiple-channel cochlear prostheses permit substantially better speech recognition than do single-channel prostheses (Gantz et al. 1988; Cohen et al. 1993; Fishman et al. 1997), indicating that multiple-channel stimulation enhances transmission of speech-related information. Nevertheless, tests of speech recognition indicate that performance is not improved by increasing the number of activated channels beyond seven in quiet listening conditions (Fishman et al. 1997; Friesen et al. 2001) or beyond ten in the presence of background noise (Friesen et al. 2001). That finding suggests that the number of functionally independent channels often is less than the number of stimulated channels.

The earliest multichannel prostheses stimulated multiple electrodes simultaneously using continuous analog waveforms. The Compressed Analog (CA) strategy, for instance, processed sound with a bank of bandpass filters, compressed the filter outputs in amplitude to reduce the dynamic range, and then 
presented all the filter outputs simultaneously to multiple implant electrodes, one filter per electrode. A similar approach is used in the more recent Simultaneous Analog Signal (SAS) strategy (ZimmermanPhillips and Murad 1999). Simultaneous stimulation of two or more nearby implant electrodes produces vector summation of electrical current fields, thereby resulting in functional interaction among channels. Wilson et al. (1991) developed a pulsatile stimulation strategy (Continuous Interleaved Sampling; CIS) that was intended to eliminate such channel interaction. In that strategy, each electrode was stimulated with an amplitude-modulated train of electrical pulses. Pulse trains on multiple electrodes were interleaved in time so that no two electrodes received simultaneous electrical currents. Most patients showed substantial improvement in speech recognition using the nonsimultaneous pulsatile CIS strategy compared with the simultaneous analog CA strategy (Wilson et al. 1991). Many contemporary speech processors for cochlear implants employ some form of interleaved pulsatile stimulation, although the SAS strategy is favored by some patients (Battmer et al. 1999; Osberger and Fisher 1999).

One interpretation of the success of patients in using an interleaved pulsatile strategy is that it eliminates direct electrical summation among multiple electrodes and reduces or eliminates interaction among the stimulated cochlear neural populations. In that way, the strategy increases the number of effectively independent channels of information that are transmitted to the brain. Nevertheless, CA, SAS, and CIS stimulation strategies have several other fundamental differences in addition to simultaneous versus nonsimultaneous stimulation, including continuous versus pulsatile waveforms and slower versus faster stimulation rates. For that reason, improved performance in the CIS strategy might not be attributable entirely to the nonsimultaneous stimulation. Moreover, several psychophysical studies have demonstrated that two or more channels, even when stimulated in an interleaved pattern, can show substantial interaction in regard to listeners' reports of pitch (Townshend et al. 1987; McDermott and McKay 1994; McKay and McDermott 1996) or loudness (Shannon 1983; McKay et al. 2001). Therefore elimination of direct electrical summation does not entirely eliminate channel interaction.

We tested the hypothesis that increasing the temporal separation of stimulus pulses on multiple cochlear implant electrodes reduces interactions among channels. The design of clinical speech processors does not permit simple comparisons in patients of pulsatile stimulation in simultaneous versus nonsimultaneous configurations, nor have there been parametric studies in patients of the influence of interchannel timing on channel interaction. For that reason, we developed an animal model in which to examine channel interactions parametrically at a basic physiological level (Bierer and Middlebrooks 2002; Middlebrooks and Bierer 2002). We chose to monitor responses to cochlear stimulation by recording from the auditory cortex. The auditory cortex is arguably not the best place to study detailed biophysical mechanisms of channel interaction inasmuch as it is many synaptic levels removed from the site of electrical stimulation; auditory nerve recordings might be better suited for detailed biophysics. Nevertheless, study of the cortex offers several advantages. Cortical responses reflect the integrated activity of the ascending auditory pathway, thereby providing a sample of the neural activity that reaches the forebrain and likely contributes to an animal's perception and behavior. Also, the straightforward tonotopic organization of the cortex aids in placing a recording site near the representation of the lowestthreshold site for any particular cochlear stimulus; in contrast, the tonotopy of the auditory nerve is less accessible, particularly in a deafened ear. Finally, electrical artifacts from cochlear stimulation complicate auditory nerve recordings, whereas cortical recordings are displaced from the electrical artifact by greater physical distance and by longer latency in the neural pathway.

We implanted anesthetized guinea pigs with arrays of six intracochlear electrodes and recorded neural spike activity from the auditory cortex with 16-channel recording probes oriented along the cortical tonotopic axis. The dependent variable was the cochlear current threshold that produced just-detectable elevation of cortical spike rates as determined by Receiver Operating Characteristic (ROC) analysis. We tested pairs of stimulated channels that varied in interchannel delay from $0 \mu$ s (i.e., simultaneous) to $2000 \mu \mathrm{s}$. We also tested the influence of spatial separation of electrodes and compared monopolar, bipolar, and tripolar electrode configurations.

As expected, interaction between implant channels generally was greatest when pulses were presented simultaneously on two channels, although there were exceptions in bipolar and tripolar conditions. Nevertheless, a single subthreshold pulse could have appreciable influence on the response to a pulse that trailed in time by up to $640 \mu$ s or more. The spatial and temporal spread of channel interaction varied considerably with electrode configuration.

\section{METHODS}

The basic procedures for cochlear stimulus presentation and multichannel cortical recording were 
similar to those in our previous work (Bierer and Middlebrooks 2002).

\section{Anesthesia and surgery}

Data were collected from 8 healthy adult pigmented guinea pigs (500-900 g). In each animal, intracochlear deafening, cochlear implantation, and cortical recordings were performed in one session lasting up to $20 \mathrm{~h}$. Animals were anesthetized initially with an intramuscular injection of ketamine hydrochloride $(40 \mathrm{mg} / \mathrm{kg})$ and xylazine $(10 \mathrm{mg} / \mathrm{kg})$. Supplementary injections of a 9:1 mixture of ketamine and xylazine were used to maintain an areflexive state. The left ear was deafened by withdrawing perilymph from the basal cochlear turn, then infusing a $10 \%$ solution of neomycin sulfate into the scala tympani; more than 2 $\mathrm{h}$ passed between neomycin infusion and the beginning of data collection. We did not routinely test for residual hearing, but in our experience with other guinea pigs, that dose of neomycin eliminates soundevoked auditory brainstem responses within a few minutes after treatment. Also, Nuttall et al. (1977) demonstrated that perilymphatic perfusion of a much lower concentration of neomycin sulfate $(10 \mathrm{mM}$; $\sim 0.6 \%$ ) eliminated sound-evoked cochlear microphonics within $60 \mathrm{~min}$.

A 6-electrode intracochlear array (Cochlear Corporation, Englewood, $\mathrm{CO}$ ) was inserted into the scala tympani of the left ear through a cochleostomy. The electrodes were platinum-iridium bands centered at $750-\mu \mathrm{m}$ intervals and were numbered $1-6$ from base to apex. A ground wire was placed in a neck muscle. The right auditory cortex was exposed, a multielectrode recording probe (described below) was inserted with reference to surface landmarks, and the cortical surface was covered with agarose $(20 \mathrm{mg}$ agarose $/ \mathrm{ml}$ Ringers solution).

All procedures were in accordance with the policies of the University of Michigan University Committee on Use and Care of Animals.

\section{Stimulus generation}

Experiments were controlled by a personal computer interfaced with Tucker-Davis hardware (Tucker-Davis Technologies, Gainesville, FL). Stimuli were controlled using custom software running in MATLAB (Mathworks Inc., Natick, MA). The signal to each active or return electrode was generated by one channel of an 8-channel digital-to analog converter (TDT DA8). Each channel was coupled to an intracortical electrode through an independent, custom-made, optically isolated current source with capacitancecoupled output. Experiments were conducted in a sound-attenuating chamber.
Stimuli consisted of single biphasic, charge-balanced pulses. Phase durations were $80 \mu \mathrm{s} /$ phase. Pulses on active electrodes were initially cathodal except when stated otherwise. Three electrode configurations of the electrical stimulus were employed. In the monopolar configuration (MP), the active electrode was a single intrascalar electrode and the return was a wire positioned in a neck muscle. In the bipolar configuration (BP), the active electrode was one intrascalar electrode and the return was the adjacent, more apical intrascalar electrode. That configuration was referred to as $\mathrm{BP}+0$ in our previous report (Bierer and Middlebrooks 2002), and for simplicity is referred to here as BP. In the tripolar configuration (TP), the active electrode was a single intrascalar electrode and the return consisted of the two adjacent electrodes (one on each side of the active electrode), each carrying half of the return current. Electrical field models and physical measurements predict that stimuli presented at a constant current level would produce increasingly more diffuse electrical fields as configurations were varied from TP to BP to MP (Kral et al. 1998; Jolly et al. 1996; Spelman et al. 1995). Absolute current levels are expressed as peak current in decibels $(\mathrm{dB})$ relative to $1 \mathrm{~mA}$.

A stimulus channel in this report refers to a signal pathway originating in the digital-to-analog converter and terminating in an active electrode and its complement of return electrode(s). Channel number corresponds to the number of the active electrode; BP channel 2, for instance, consists of active electrode 2 and more apical return electrode 3 . On a given trial, either one or two cochlear implant channels were stimulated. In this study, the more apical channel always was channel 5, and the more basal channel was either channel 2 or 3, as indicated. The separation between active electrodes was 1.5 or $2.25 \mathrm{~mm}$ when the basal channel was channel 3 or 2 , respectively. In two-channel conditions, the same electrode configuration was used on both channels.

Current levels were varied over a range from below threshold to 5 to $15 \mathrm{~dB}$ above threshold in either 1- or 2-dB steps. In each block of trials, electrode configurations were held constant, and stimulus current level, active electrode separation, and temporal offset were varied among trials. Every combination of current level, active electrode separation, and temporal offset was presented once in random order, then every combination was repeated in a different random order until each stimulus combination was tested 10 times.

Interchannel temporal offsets were expressed as the time from the onset of the first pulse to the onset of the second pulse, as shown in Figure 1. 

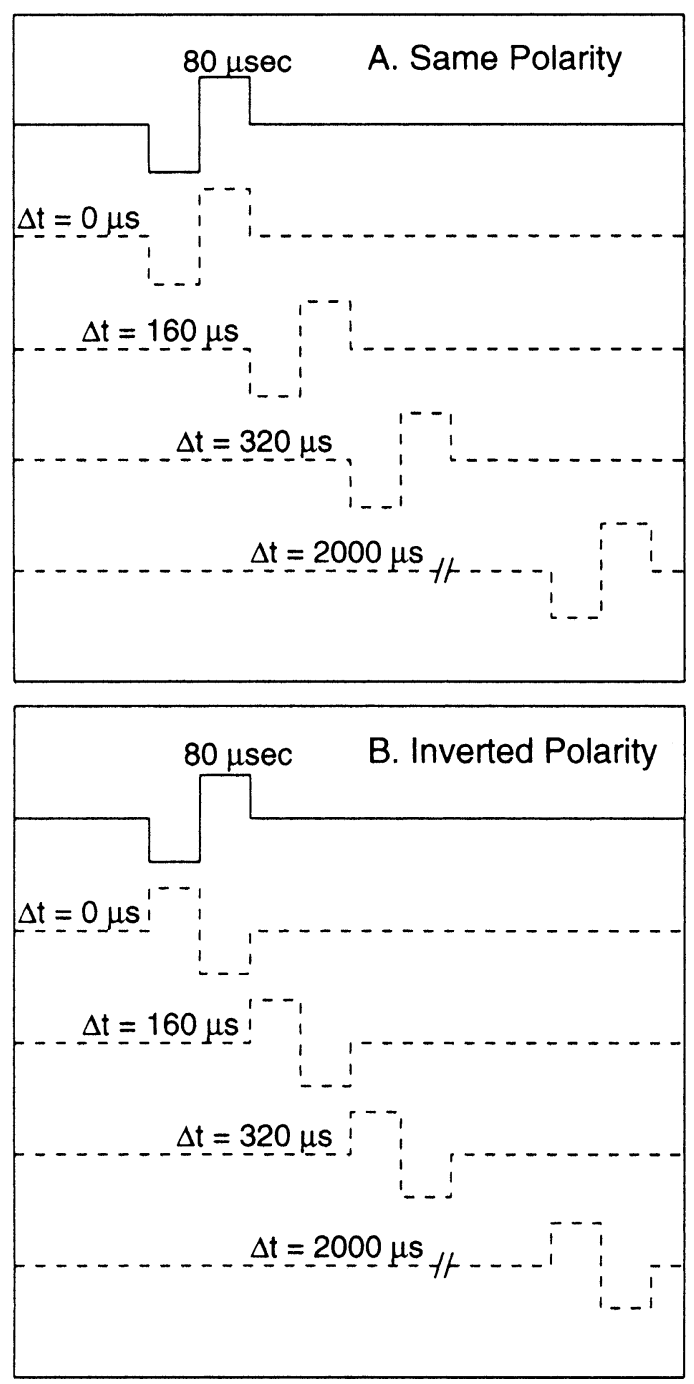

FIG. 1. Schematics of two-channel stimuli. Each channel was stimulated with a biphasic pulse of duration $80 \mu \mathrm{s} /$ phase, represented by the solid line in each panel. The broken lines represent the stimulus on the more apical channel. Temporal offsets were measured from the beginning of the first pulse to the beginning of the second. In each panel, temporal offsets of $0,160,320$, and $2000 \mu \mathrm{s}$, respectively, are shown. Top and bottom panels represent the sameand inverted-polarity conditions.

Temporal offsets ranged from 0 (simultaneous) to $2000 \mu$ s. We chose $2000 \mu$ s as the longest temporal offset because that is the longest offset that is possible given two channels stimulated at 250 pulses/s, as in the Spectral Peak (SPEAK) speech-processing strategy (Skinner et al. 1994). Same-phase stimulation of two channels indicates that pulses on both active electrodes were presented with the cathodic phase first (left panels of Fig. 1), whereas invertedphase stimulation indicates that the pulse on one active electrode was initially anodic and the pulse on the other active electrode was initially cathodic (right panels of Fig. 1).

\section{Multichannel recording and spike sorting}

Cortical activity was recorded with silicon-substrate multichannel recording probes (Center for Neural Communication Technology, Ann Arbor, MI; Drake et al. 1988; Najafi et al. 1985). Each recording probe had 16 recording sites along a single shank at intervals of $100 \mu \mathrm{m}$ (center to center). The shank was 15 $\mu \mathrm{m}$ thick and $100 \mu \mathrm{m}$ wide, tapering in width from $100 \mu \mathrm{m}$ to $15 \mu \mathrm{m}$ over the segment containing the recording sites. The multichannel recording probe permitted recording of spike activity simultaneously from 16 cortical sites.

Recording probes penetrated the primary auditory cortex in the right hemisphere, from caudodorsal to rostroventral and roughly parallel to the cortical surface. We attempted to position the probe in the middle cortical layers, aligned with the cochleotopic gradient along which the representation of cochlear place of stimulation changes most rapidly. In our previous study in which we used these probes to study cortical responses to tones, the 16 recording sites typically spanned about 2-3 octaves of the tonotopic frequency representation (Arenberg et al. 2000). In the guinea pig area A1, neurons sensitive to basal cochlea stimulation (high frequencies) are situated dorsocaudally, and apical cochlea (low frequencies) are situated ventrorostrally (Hellweg et al. 1977; Redies et al. 1989; Arenberg et al. 2000; Wallace et al. 2000; Bierer and Middlebrooks 2002). Prior to detailed study at each probe position, tuning properties of rostral and caudal cortical sites were estimated by observing responses to BP stimuli on the most apical and basal stimulus channels. If the reverse cochleotopic order was detected, indicative of the dorsocaudal field (area DC; Redies et al. 1989), the probe was retracted and placed further ventral and rostral in area A1.

Signals measured from the recording probe were amplified with a custom 16-channel amplifier, digitized at a $25-\mathrm{kHz}$ rate, filtered, and then stored on the computer hard disk. Unit activity was isolated from the digitized signal offline using custom spike-sorting software (Furukawa et al. 2000). Spike times were stored at $20-\mu$ s resolution for further analysis. We sometimes encountered well-isolated single units, but most recordings were of unresolved clusters of a small number of units.

\section{Data analysis}

Detailed measurements of cortical activation patterns were obtained from one probe placement in each of 8 guinea pigs. At each probe placement, stable recordings of single and multiunit clusters were obtained at 16 recording sites for a total of 128 recording sites. 

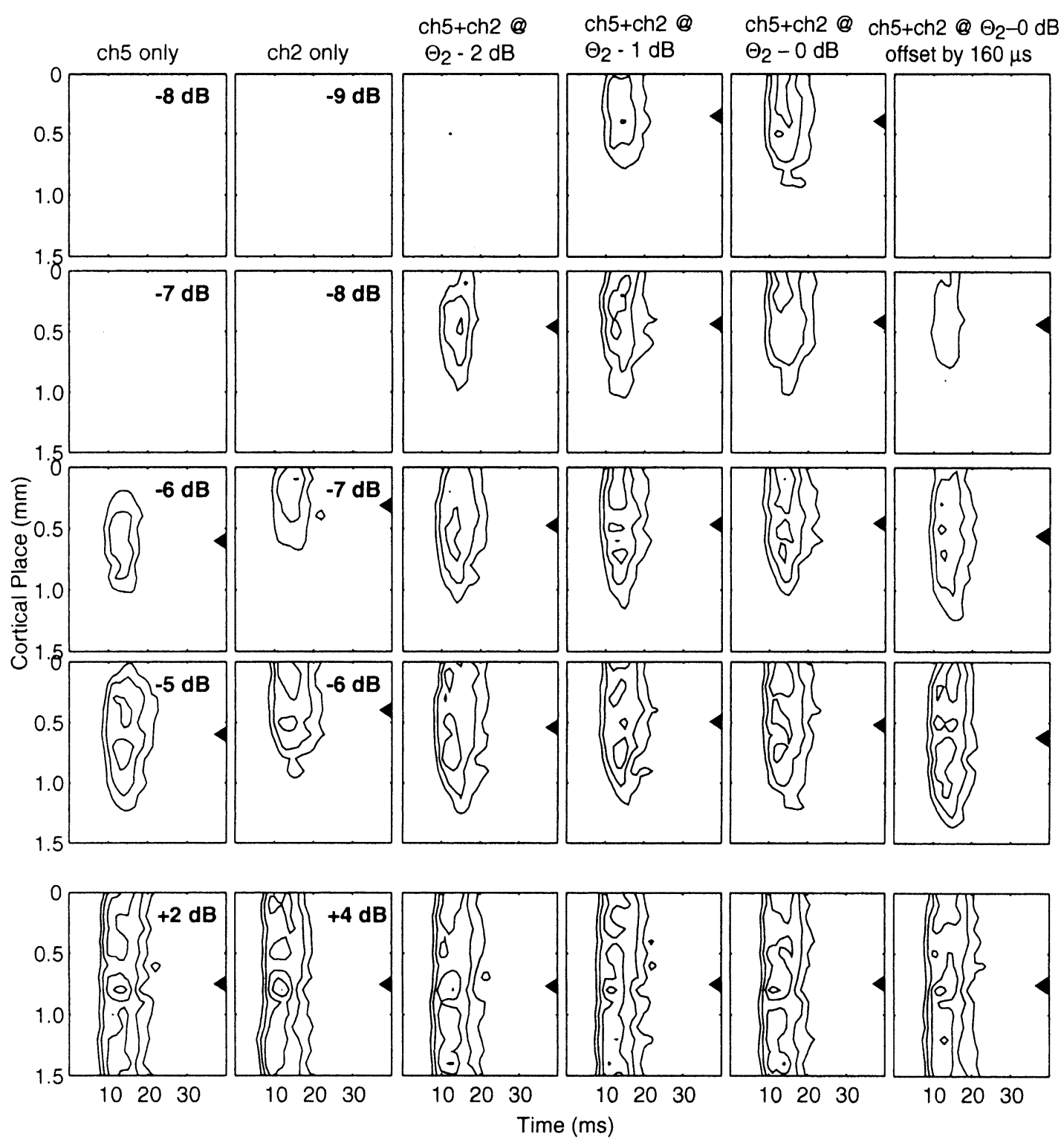

FIG. 2. Cortical images of one- and two-channel stimuli. Each panel represents the cortical image of one stimulus averaged across 10 trials. The left two columns represent responses to single-channel stimulation of channels 5 (first column) and 2 (second column). Current levels are expressed in $\mathrm{dB}$ re: $1 \mathrm{~mA}$. Columns 3, 4, and 5 represent two-channel stimulation in the simultaneous condition with the current level on channel 2 fixed at 2, 1 , and $0 \mathrm{~dB}$, respectively, below the threshold for channel 2 alone (i.e., $\Theta_{2}-2$, $\Theta_{2}-1$, and $\Theta_{2}$ ). The rightmost column represents a condition in

Threshold current levels based on cortical spike counts were determined by using procedures from Signal Detection Theory (Green and Swetts 1966). In conditions in which only one channel was stimulated, ROC curves were computed from spike counts obtained on trials in which a stimulus was or was not present. In two-channel stimulation conditions, a fixed current level was present on one channel on all trials, and the ROC curve was computed from trials in which a stimulus on the second channel was or was which the stimulus level on channel 2 was fixed at $\Theta_{2}$ with a temporal offset of $160 \mu \mathrm{s}$. For each cortical image, the abscissa represents poststimulus time and the ordinate represents cortical place relative to the most caudal recording site. Contours represent mean spike counts expressed as percent of the maximum count on each cortical channel; contours are drawn at 20, 40,60, and 80 of the maximum count. Triangles to the right of each panel represent the centroid locations. Data are from animal GP02.

not present. The area under an ROC curve was converted to a discrimination index $\left(d^{\prime}\right)$. Values of $d^{\prime}$ were computed for current levels tested in 1- or 2-dB steps. Linear interpolation was used to estimate the current level corresponding to $d^{\prime}=1$, which was taken as the threshold. We regard this as a fairly conservative estimate of threshold, compared to analysis of simple mean spike rates, inasmuch as the ROC analysis incorporated the trial-by-trial variability of spike counts on stimulus and nonstimulus trials. We 
designate the threshold for channel $i$ by $\Theta_{i}$. Channel interactions were quantified by threshold shifts, $\Delta \Theta$, which designate the changes in the threshold for channels $i$ resulting from a constant-level stimulus on channel $j$. Threshold shifts were expressed in $\mathrm{dB}$ as the channel $i$ threshold in the presence of a channel $j$ stimulus minus the threshold in the absence of channel $j: \Delta \Theta=\Theta_{i+j}-\Theta_{i}$. Negative threshold shifts designate reductions in channel $i$ thresholds resulting from stimulation of channel $j$.

The cortical image of any particular stimulus (as in Fig. 2) was represented by the distribution of stimulus-driven cortical activity across all recording sites and across poststimulus time (Bierer and Middlebrooks 2002). Cortical images were derived from simultaneous recordings at 16 cortical sites averaged across 10 trials. For the purpose of computing cortical images, spike rates were normalized at each recording site. That was accomplished by computing at each recording site the mean spike rate for each condition of stimulus channel, current level, and temporal offset, then taking the 5th and 95th percentile of the distribution of mean spike rates as the spontaneous rate and maximum rate, respectively. The range of spike rates between those rates was used to compute a normalization factor for each site. That normalization method emphasized stimulus-driven changes in activity rather than absolute spike numbers across channels. Cortical images are illustrated using contour plots (Fig. 2), which were drawn using the contour function in MATLAB. As was done previously, the centroid of the cortical image was defined as the normalized-spike-rate-weighted center of mass calculated from all the sites at which the firing rate was above threshold; the centroid computation collapsed spike rates across all time bins (Bierer and Middlebrooks 2002).

Statistical comparisons of threshold shifts between pairs of conditions were made using a nonparametric sign test. The nonparametric test was chosen because in many cases the threshold shift in one of a pair of conditions was so large that the magnitude of the shift could not be determined, even though the lower bound of the shift was measured to be larger than the threshold shift measured in the other condition.

\section{RESULTS}

We refer to the characteristic spatiotemporal distribution of cortical spike activity elicited by a particular stimulus at one particular level as the cortical image of that stimulus. We begin by describing the cortical images of one- and two-channel cochlear implant stimuli. Then we describe spike-rate-versus-currentlevel functions, showing that sub-threshold stimula- tion of one channel could reduce the threshold for a second channel. We evaluate these threshold shifts at various cortical sites relative to the tonotopic representations of the two stimulus channels. Finally, we test the sensitivity of threshold shifts to the relative cochlear spacing, temporal offset, and relative polarity of two-channel stimuli.

\section{Cortical images of one- and two-channel stimuli}

Figure 2 presents examples of cortical images of oneand two-channel cochlear implant stimuli in the bipolar (BP) electrode configuration. The vertical columns of panels represent, from left to right, the cortical images of stimuli presented as follows: (1) to channel 5 alone; (2) to channel 2 alone; (3) to channel 5 with channel 2 stimulated simultaneously at $\Theta_{2}-2 \mathrm{~dB}$; (4) to channel 5 with channel 2 stimulated simultaneously at $\Theta_{2}-1 \mathrm{~dB}$; (5) to channel 5 with channel 2 stimulated simultaneously at $\Theta_{2} \mathrm{~dB}$; and (6) to channel 5 with channel 2 stimulated at $\Theta_{2}$ $\mathrm{dB}$ with a $160-\mu$ s temporal offset. Each horizontal row of panels represents the response at a fixed current level on channel 5, except for the second column, which shows responses to stimulation of channel 2 alone. In each panel, the vertical axis represents the location along the cortical recording probe relative to the most caudal recording site, the horizontal axis shows time after the stimulus onset, and the contours represent the normalized spike rate at each recording site, increasing from $20 \%$ to $80 \%$ of maximum activity in steps of $20 \%$. Single-channel stimulation produced spatially restricted foci of cortical activity at threshold levels (third row, left two columns). Centroids of the cortical images of near-threshold stimuli on channels 5 and 2 were located at 602 and $314 \mu \mathrm{m}$, respectively. The relative locations of those centroids were consistent with the known cochleotopic organization of the primary auditory cortex of the guinea pig in that the cortical image of the more apical electrode pair (channel 5) was located further rostral than that of the basal electrode pair (channel 2; Bierer and Middlebrooks 2002). As the current level was increased to the highest tested levels (bottom row), cortical images broadened in cortical extent to cover the entire recording array.

The stimulus current levels on channel 5 were constant across each row of cortical images in Figure 2 (except for column 2, in which channel 5 was not stimulated). When channels 2 and 5 were stimulated simultaneously (columns 3, 4, and 5), robust cortical responses were elicited at levels $2 \mathrm{~dB}$ or more below the thresholds for each channel stimulated alone. At a near-threshold channel 5 level (i.e., row 3 ), addition of a pulse on channel 2 at $\Theta_{2}-2 \mathrm{~dB}$ produced a cortical image that encompassed both of the cortical 


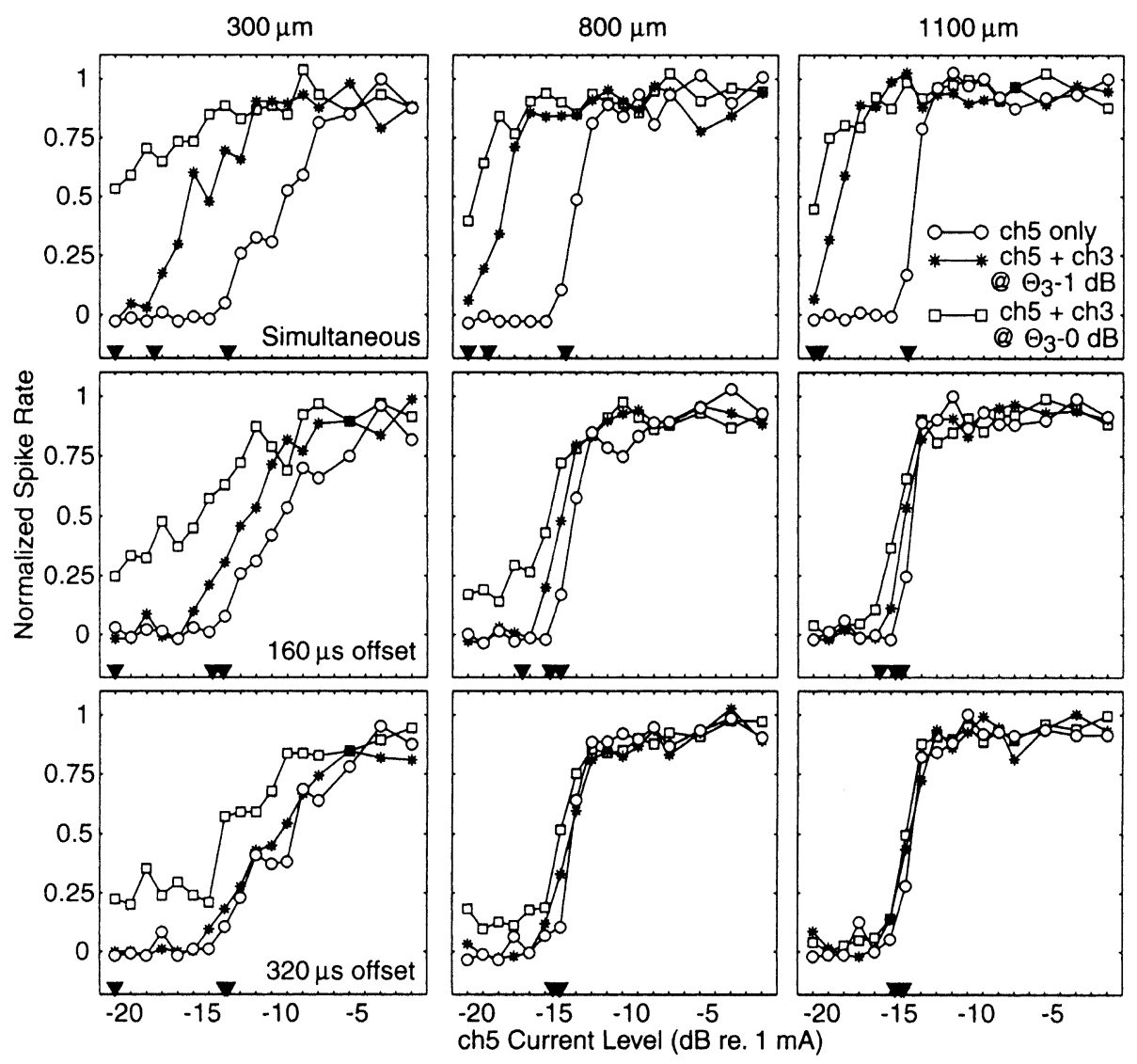

FIG. 3. Rate-versus-level functions for three recording sites and three temporal offsets. Each column of panels represents responses obtained from one recording site, 300, 800, or $1100 \mu \mathrm{m}$ relative to the most caudal site. Rows of panels represent responses to simultaneous (top), $160-\mu$ s offset (middle) and 320- $\mu$ s offset (bottom). For each panel, the abscissa represents the stimulus current level delivered to channel 5 and the ordinate represents the normalized spike rate. Rate-level functions are drawn for stimulation of channel 5 alone (circles) or with the current level on channel 3 constant at its threshold of $-6 \mathrm{~dB}$ re: $1 \mathrm{~mA}$ (squares) or constant at $-7 \mathrm{~dB}$ re: $1 \mathrm{~mA}$ (asterisks). Filled triangles indicate threshold current levels in the one- and two-channel conditions. Data are from animal GP43. areas that were activated by each channel individually. The centroid for low-level simultaneous stimulation of channels 2 and 5 was located between the centroids of the two single-channel centroids.

When stimulation of channel 2 preceded stimulation of channel 5 by $160 \mu$ s (rightmost column), cortical responses were elicited at the second level shown, about $1 \mathrm{~dB}$ below the threshold for stimulation of channel 5 alone. That observation indicates that the influence of channel 2 on channel 5 persisted for temporal offsets at least as long as $160 \mu \mathrm{s}$ after the offset of the channel 5 stimulus.

In summary, the differences between two-channel cortical images and the constituent single-channel stimuli include (1) a reduction of cortical response threshold for simultaneous or nonsimultaneous twochannel stimulation, (2) a shift of the cortical centroid of activity to a location intermediate to the centroids of activity elicited by the two single-channel stimuli, and (3) an extent of cortical activation greater than the area encompassed by the responses to each of the two single-channel stimuli individually.

\section{Threshold shifts}

Spike rates at nearly every recording site increased monotonically or increased to a plateau as current levels were increased. Figure 3 shows examples of spike-rate-versus-current-level functions at three recording sites, 300, 800, and $1100 \mu \mathrm{m}$ relative to the caudalmost site on the recording probe. Each column of panels represents one recording site, and top, middle, and bottom rows of panels represent temporal offsets of 0,160 , and $320 \mu$ s, respectively. Lines marked with circles indicate rate-level functions obtained with stimulation of channel 5 alone; within each column, the differences among rows of panels in the channel-5-alone rate-level functions demonstrate variability among repeated measurements. The threshold for stimulation of channel 3 alone, $\Theta_{3}$, was determined as described in the Data Analysis subsection. The lines marked with squares or asterisks represent the rate-level functions obtained for stimulation of channel 5 while the level on channel 3 was held constant at $\Theta_{3}$ or $\Theta_{3}-1 \mathrm{~dB}$, respectively. In every panel in the figure, stimulation of channel 3 increased the spike rate in response to the channel 5 stimulus, with the effect of displacing rate-level functions to the left. Filled arrowheads on the abscissa indicate thresholds determined for the channel-5-alone and two-channel conditions $\left(\Theta_{5}\right.$ and $\Theta_{5+3}$, respectively). We designate a reduction in current threshold for channel 5 resulting from addition of the channel 3 stimulus by a negative threshold 



FIG. 5. Differences between threshold shifts measured at the channel 2 or channel 3 centroid compared with that measured at the channel 5 centroid. Points falling above the positive diagonal indicate threshold shifts were greater for sites near the channel 2 or channel 3 centroid. The distribution is collapsed across active electrode separations of 1.5 and $2.25 \mathrm{~mm}$.

whereas there was no little or no threshold shift recorded at rostral sites. In the TP case, a threshold shift was recorded only at the most caudal sites. Data points are missing in the TP condition at sites at which the cortical response was too weak to determine a reliable threshold; this is expected from the restricted cortical images of TP stimuli (Bierer and Middlebrooks 2002). The magnitude of threshold shifts in the TP configuration was rather variable across animals but was negligible in many cases.

In the illustrated example, addition of a temporal offset of 160 or $320 \mu$ s essentially abolished the threshold shifts in the BP and TP configurations. In the MP configuration, threshold shifts in the presence of temporal offsets were restricted to cortical sites near the centroid of the cortical image of channel-2-alone stimuli (indicated by triangles on the abscissas).

The example illustrated in Figure 4 is representative of all eight cases in the following respects: The largest threshold shifts were observed in the simultaneous MP configuration. In the nonsimultaneous MP conditions, the magnitude of threshold shifts tended to decrease with increasing temporal offset. The dependence on temporal offset was more variable in the BP and TP configurations, sometimes showing little or no shift at any temporal offset (see the following subsection). Within each of the simultaneous MP and BP conditions, the largest threshold shifts tended to be recorded at cortical sites near the centroid of the cortical image of the near-threshold fixed-level stimulus, in this case channel 2. Threshold shifts in the TP configuration generally were smaller than for MP or BP and were entirely absent in some cases.

Shifts in threshold tended to be greatest in magnitude at cortical sites near the centroid of the cortical image of the near-threshold fixed-level channel (channel 2 or 3 in the previous examples). That can be seen in the previous example (shown in Fig. 4), in which the triangles along the abscissa indicated the locations of cortical centroids for channels 2 and 5 . Figure 5 shows examples of the threshold shift measured near the centroid of the fixed-level channel compared with that near the channel for which the threshold was measured (e.g., channels 2 and 5, respectively, for the examples shown in Figs. 3 and 4). Data are shown for the condition in which the current on channel 2 or 3 was fixed at $1 \mathrm{~dB}$ below the threshold for that channel. Threshold shifts, collapsed across MP, BP, and TP configurations, were significantly greater in magnitude at the cortical centroid of the fixed-level channel than at the centroid of the channel for which thresholds were measured; that comparison was significant $(p<0.005)$ for temporal offsets of 0,160 , and $320 \mu \mathrm{s}$, but not significant for $640 \mu$ s (data not shown), both with the more basal channel or the more apical channel stimulated at a fixed level. That result implies that a subthreshold pulse at a particular cochlear site increased the sensitivity at that site to a simultaneous or later pulse presented at another cochlear site.

We anticipated that threshold shifts would be sensitive to the separation between the two active intracochlear electrodes. That hypothesis was difficult to 


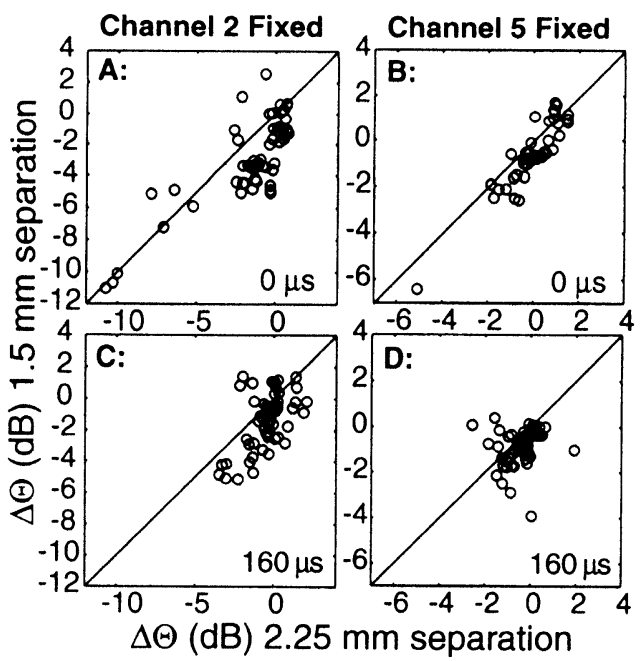

FIG. 6. Threshold shifts for two BP channel separations. Each panel shows the threshold shifts measured in response to two-channel stimulation with a channel separation of $2.25 \mathrm{~mm}$ compared with that of $1.5 \mathrm{~mm}$. Symbols represent every recording channel in which thresholds could be computed for both separations (i.e., 8 animals, as many as 16 channels per subject). The left column of panels shows threshold shifts in the condition in which the current on the more basal channel (i.e., channel 2 or 3 ) was fixed at $1 \mathrm{~dB}$ below its threshold, and the right column of panels shows threshold shifts for the condition in which the current on the more apical channel was fixed. Upper and lower rows of panels represent simultaneous and $160-\mu$ s offset conditions. Points falling below the positive diagonal indicate threshold shifts that were greater for smaller active electrode separations.

test in this study because of the limited number of active electrode separations that could be implemented on a 6-electrode cochlear implant; for instance, only 2 nonoverlapping TP channels could be implemented. Figure 6 compares the threshold shifts for 1.5 and 2.25-mm cochlear active electrode separations in the BP electrode configuration; left and right columns of panels show conditions in which the more basal channel (left) or more apical channel (right) was fixed at a near-threshold level. In the BP configuration, threshold shifts were significantly greater in magnitude $(p<0.001)$ for 1.5 - than for 2.25 $\mathrm{mm}$ active electrode separations; the difference was significant for all temporal offsets from 0 to $640 \mu$ s and for conditions in which either the more basal or more apical channel was fixed in level. The MP configuration showed no consistent difference between 1.5- and 2.25-mm active electrode separations for either temporal offset (data not shown), presumably because there was so much overlap in electrical fields between pairs of electrodes. These findings indicate that channel interaction with the BP configuration is influenced by the distance between the two active electrodes of the two-channel stimuli, whereas channel interaction with the MP configuration was great for both tested active electrode separations.
Temporal offset between two channels of stimulation

As shown in the previous examples, threshold shifts most often decreased with increasing temporal offset between pulses on two channels. Figure 7 shows threshold shifts as a function of temporal offset for 8 animals and 3 electrode configurations. Thresholds were measured for channel 5 in the presence of a stimulus on channel 2 or 3 fixed at $1 \mathrm{~dB}$ below the channel 2 or channel 3 threshold; the plotted threshold shifts were measured at the centroid for channel 2 or 3 . Threshold shifts observed for simultaneous and nonsimultaneous conditions are shown with filled and open symbols, respectively. In the MP configuration, threshold shifts consistently were largest in the simultaneous condition; they were rather variable in the simultaneous $\mathrm{BP}$ and $\mathrm{TP}$ conditions. Threshold shifts in the nonsimultaneous conditions generally declined with increases in the temporal offset. Data from the MP configuration showed a roughly logarithmic dependence, declining at a rate of $0.5 \mathrm{~dB}$ per doubling of temporal offset (range across 7 animals: 0.3-1.0). Again, the results were more variable for the $\mathrm{BP}$ and $\mathrm{TP}$ configurations. A subthreshold pulse on one channel in some cases lowered the threshold of a pulse on a second channel that followed in time by up to $640 \mu \mathrm{s}$.

The data presented above represent conditions in which thresholds were measured for a test pulse that was simultaneous with or was preceded by a nearthreshold fixed-level pulse. We also tested conditions in which the fixed-level stimulus followed the test pulse for which the threshold was measured. Figure 8 compares threshold shifts under conditions in which the fixed-level channel led or trailed the channel for which the threshold shift was measured. The three panels show data for temporal offsets of 160,640 , and $2000 \mu$ s. Data are combined across all animals, recording sites, electrode configurations, active electrode separations, and fixed levels $\left(\Theta_{2,3}-1\right.$ and $\Theta_{2,3}-2 \mathrm{~dB}$ ). For temporal offsets up to $640 \mu \mathrm{s}$, there was a small but significant tendency for threshold shifts to be larger when the fixed-level channel was first (mean differences generally $<1 \mathrm{~dB} ; p<0.001$ for all conditions except for $640-\mu$ s offset with the more apical channel fixed: $p>0.05)$. The effect of temporal order was reversed for longer temporal offsets. A near-threshold pulse on one channel tended to elevate the threshold of a pulse on a second channel that followed by $2000 \mu \mathrm{s}(p<0.001)$.

\section{Polarity inversion between channels}

We tested in four animals a condition in which the polarity of the electrical current was inverted between 


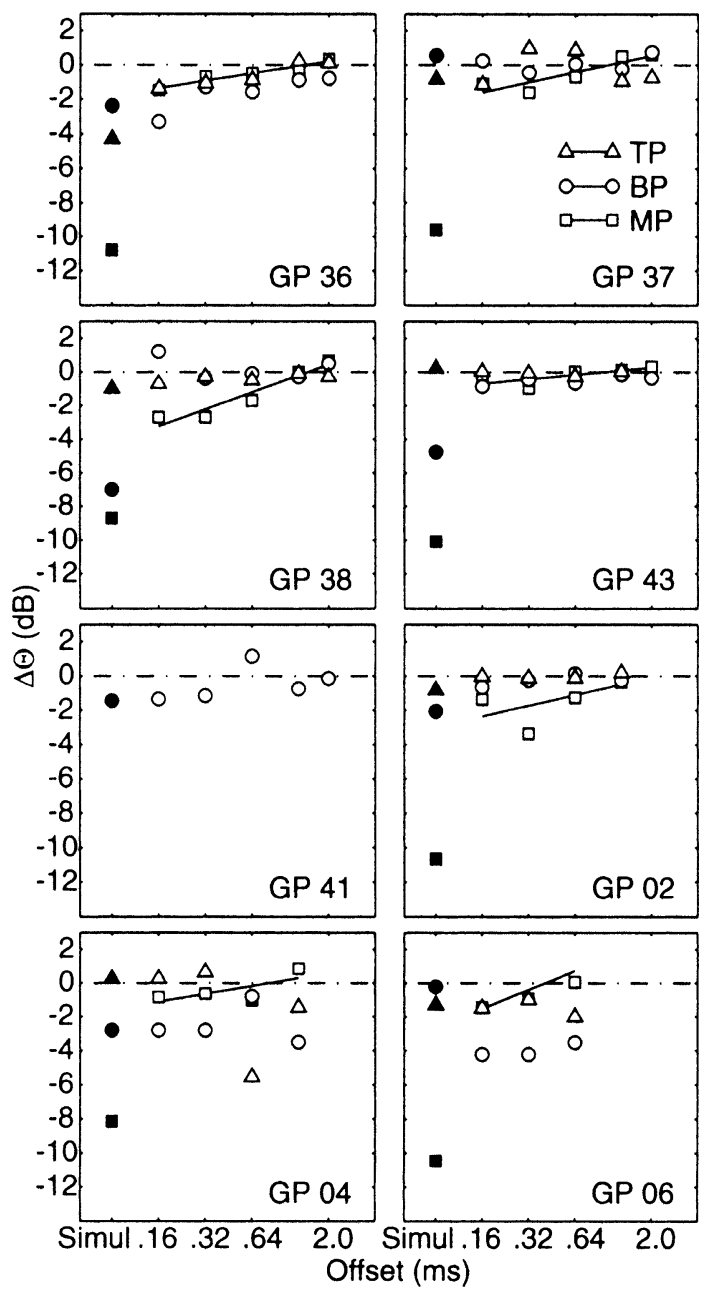

FIG. 7. Threshold shifts as a function of temporal offset. Each panel shows data from one animal, indicated by the number in the lower right corner. Symbol shapes represent electrode configuration. The filled symbols represent responses to the simultaneous condition. The open symbols represent threshold shifts measured in response to varying temporal offsets from 160 to $2000 \mu$ s. The lines show the least-squared fits to the nonsimultaneous MP data (open squares). In each case, threshold shifts were measured the cortical site closest to the fixed-level (channel 2 or 3) centroid.

the two stimulus channels. That is, in the invertedpolarity condition, the biphasic pulse on electrode 5 was initially cathodal and the biphasic pulse on electrode 2 or 3 was initially anodal. Examples from one representative animal are shown in Figure 9. The inverted-polarity simultaneous MP condition was unlike every other simultaneous condition in this study in that thresholds were elevated by around $4 \mathrm{~dB}$ at all cortical sites in this example. The MP condition with a $160-\mu$ s temporal offset, in contrast, showed a particularly strong threshold reduction. Other conditions in this animal produced threshold reductions of various magnitudes. The results from the illustrated example were consistent across inverted-polarity conditions in all animals in that the simultaneous MP
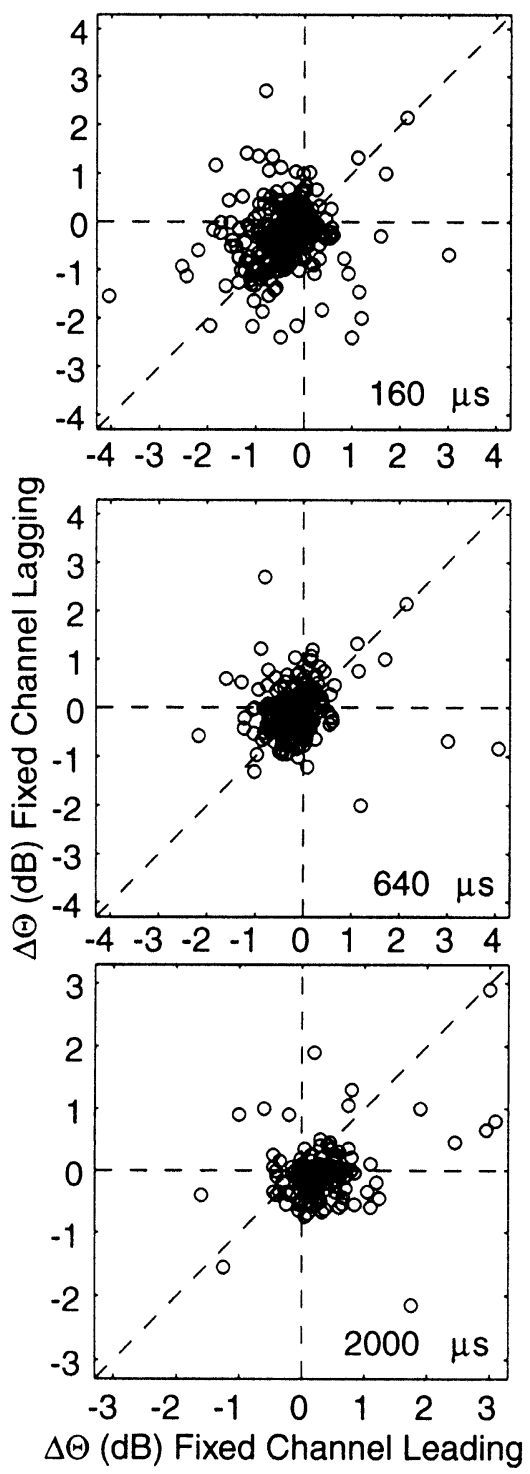

FIG. 8. Sensitivity to the order of fixed- and variable-level channels. The plots compare threshold shifts in conditions in which the pulse on the fixed-level channel led (horizontal coordinate) or lagged (vertical coordinate) the pulse on the channel that was varied in current level. Data are compiled across all animals, configurations, and active electrode separations.

condition produced a threshold elevation (range among animals, measured at the cortical centroid of the fixed-level channel: +3.2 to $+4.7 \mathrm{~dB}$, median $=4.1$ $\mathrm{dB}$ ), whereas any threshold shift in any of the other conditions was a threshold reduction. Threshold reductions in the MP 160- $\mu$ s-temporal-offset condition were most reliably large, ranging from -0.7 to -6.7 $\mathrm{dB}($ median $=-6.4 \mathrm{~dB})$. Threshold shifts in other MP conditions and all $\mathrm{BP}$ and $\mathrm{TP}$ conditions ranged among animals from +0.5 to $-4.8 \mathrm{~dB}$ (median $=0.7$ $\mathrm{dB})$. Possible mechanisms for threshold elevations and reductions in the inverted-polarity conditions are considered in the Discussion. 


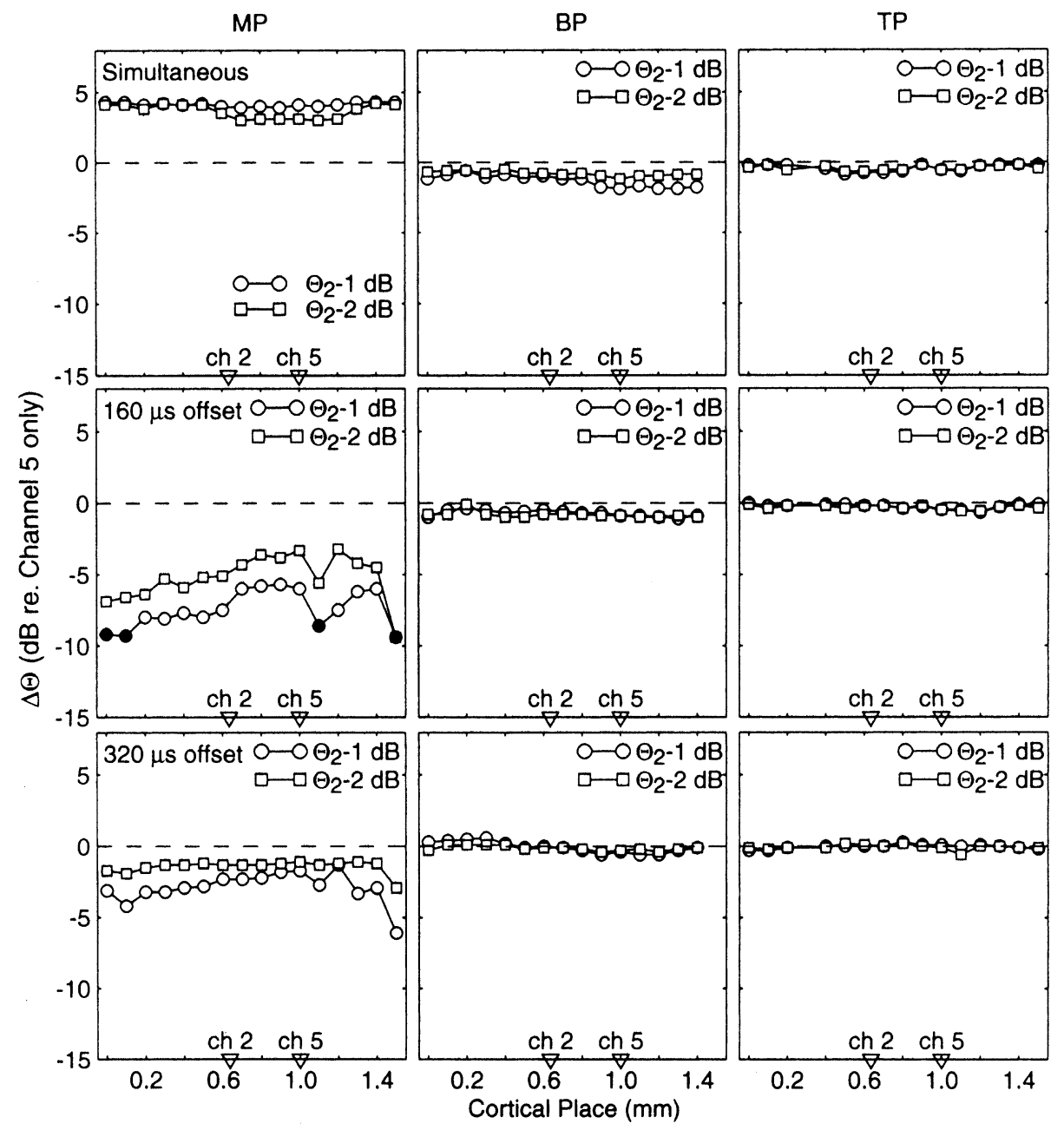

FIG. 9. Threshold shifts across cortical recording sites for invertedphase stimulation for MP, BP, and TP electrode configurations and three temporal offsets. Conventions are as in Fig. 4. Data are from animal GP38.

\section{DISCUSSION}

The results of the present study demonstrate that the current level required to elicit cortical responses by a single cochlear implant channel was influenced by the presence of threshold or subthreshold activity on a second channel. The magnitude and direction of that influence was dependent on several factors: (1) the electrode configuration, (2) the spatial separation of the two active electrodes, (3) the relative timing of the two stimuli, and (4) the relative polarity. Conversely, there was only a weak dependence in the order of channels (for temporal offsets up to $\sim 640$ $\mu \mathrm{s})$ or on the relative apical and basal locations of fixed- and varying-level channels. These results provide insight into possible mechanisms of channel interaction and have implications for the design of speech-processing strategies and/or electrode design for cochlear prostheses.

\section{Relation to previous studies}

There have been few previous physiological studies of responses to multiple cochlear stimulating elec- trodes. In one early study, Merzenich and White (1977) recorded responses of neurons in the cat inferior colliculus to stimulation of two intracochlear electrodes. When the electrodes were stimulated simultaneously, the collicular responses were substantially greater in a same-polarity condition compared with an inverted-polarity condition. Contrary to the present results, however, when pulses were delivered in phase through the two electrodes nonsimultaneously (offset by as little as $75 \mu \mathrm{s}$ ), a pulse on one electrode had no effect on the threshold for another electrode.

One can gain some understanding of responses to nonsimultaneous pulses on two nearby electrodes by examining the responses to pairs of pulses presented on a single electrode. Cartee et al. (2000) recorded cat auditory nerve responses to paired pulses. Their electrical stimuli were pseudomonophasic, in contrast to our biphasic pulses, and were presented through an electrode inserted into the internal auditory meatus, compared with our intrascalar electrodes. Paired-pulse summation was observed, meaning that a subthreshold leading pulse resulted in an increased probability of a response to a following pulse. The 
summation decreased with increasing interpulse interval with a time constant of $147 \mu \mathrm{s}$. The summation time constant appears to be shorter for a meatal stimulation site compared with an intracochlear site. Cartee et al. (2000) estimated an intracochlear summation time constant of $504 \mu$ s from data shown by Dynes (1996). The many methodological differences preclude close comparison with the present results, but summation time constants of a few hundreds of microseconds are of the same order of magnitude as the sensitivity to temporal offsets that we observed.

The present physiological results can be compared with published human psychophysical results. Relevant psychophysical results are available from studies of detection thresholds, loudness summation, and pitch perception. White et al. (1984) measured detection thresholds for pairs of biphasic pulses that were presented simultaneously, one on each of two intracochlear electrodes. They compared thresholds in same- or inverted-phase conditions as an indication of the magnitude of channel interactions. Consistent with the present physiological results, they found that channel interactions increased with increasing proximity of electrodes and were greater in a MP configuration than in a BP electrode configuration.

One common psychophysical measure of channel interaction is based on subjects' estimates of loudness (Shannon 1983; White et al. 1984; Tong and Clark 1986; McKay and McDermott 1996; McKay et al. 2001). In such procedures, subjects compare the loudness of a two-channel stimulus with that of a onechannel stimulus. That a two-channel stimulus is usually perceived as louder is referred to as loudness summation. We must use some care in comparing measures of loudness summation with our measures of threshold reduction because loudness summation involves estimates of the perceived magnitudes of suprathreshold stimuli, whereas our measurements were made around threshold. Nevertheless, studies of loudness summation provide some indication of the parameters that influence channel interactions.

Shannon (1983) tested loudness summation using simultaneously presented sinusoidal electric stimuli. He found that loudness summation tended to follow expectations based on simple vector summation of electrical fields: loudness was increased in same-polarity stimulus conditions and decreased in invertedpolarity conditions. The MP electrode configuration produced greater spatial overlap and greater loudness summation than did the BP configuration, as in the present results. White et al. (1984) tested loudness summation in response to single biphasic pulses presented nonsimultaneously, one to each of two channels. There were considerable intersubject differences, but subjects' loudness judgments generally were sensitive to interpulse temporal offsets of up to $5 \mathrm{~ms}$.
Tong and Clark (1986) tested loudness summation using interleaved pulse trains. They found that loudness tended to increase with increasing cochlear separation between active cochlear electrodes. That result is consistent with results from acoustical stimulation studies that show that loudness tends to increase with increasing separation in frequency between two tones (Plomp 1976). One interpretation of the Tong and Clark results is that the increased loudness is a consequence of increased spread of excitation in central structures resulting from increased spread of excitation in the cochlear nerve. Consistent with that view, the present physiological results showed that the cortical images of two-channel stimuli encompassed the images of either channel alone (e.g., Fig. 2). We note, however, the possibility of other models of loudness growth that do not involve spread of cochlear nerve excitation (Zeng and Turner 1991; Hellman 1994).

McKay et al. (2001) tested loudness summation using interleaved pulse trains. They found a rather complicated interaction among several factors including active electrode separation, pulse rate, stimulus level, and electrode configuration. All the loudness matches in that study were performed at the midpoint or the maximum of the dynamic range. At the lower level, but not the higher, the closest active electrode separation $(0.75 \mathrm{~mm})$ produced greater loudness summation than did $2.25-\mathrm{mm}$ or greater separations. The dependent variable in the present study was detection threshold, which may be regarded as the lowest point in the neural dynamic range. At that level, we observed greater threshold reduction with $1.5-\mathrm{mm}$ than with $2.25-\mathrm{mm}$ active electrode separations, but only in the BP configuration. Note that we could test only a limited range of electrode separations because of the limited number of electrodes in our guinea pig cochlear implant.

Channel interaction also can influence pitch perception. Pitch perception in cochlear implant studies refers to the perceptual dimension in which subjects rank successive cochlear implant channels. Both simultaneous and nonsimultaneous activation of two cochlear implant channels have been shown to elicit a single pitch that is intermediate to the pitch of the two channels stimulated individually. An intermediate pitch percept was perceived by subjects using either broad (MP) or more restricted electrode configurations (BP and common ground) (MP: Townshend et al. 1987; BP/common ground: McDermott and McKay 1994; McKay and McDermott 1996). The ratio of currents delivered to each stimulus channel influenced the location of the intermediate pitch-as the current level on channel A was increased, the intermediate pitch approached that of channel A, and vice versa for channel B (Townshend 
et al. 1987; McDermott and McKay 1994). In our study, the centroids of our cortical images varied systematically with the cochlear place of stimulation (Bierer and Middlebrooks 2002; Middlebrooks and Bierer 2002), presumably in analogy with psychophysical pitch perception. Cortical images of two-channel stimuli showed a broad single peak encompassing the images of the two component stimulus channels. That might be analogous to the intermediate pitch that is reported by human implant users.

\section{Possible mechanisms of channel interaction}

Interactions between stimulated channels are likely to take place at multiple levels of the auditory system, from the cochlea to the cortex. In the central nervous system, studies that use acoustic stimuli show interchannel interaction in the form of lateral (or twotone) inhibition. Two-tone inhibition has been demonstrated in the cochlear nucleus (e.g., Young 1998), the inferior colliculus (e.g., Ramachandran et al. 1999), and the auditory cortex (e.g., Shamma et al. 1993). In our studies, even the most restricted electrical stimuli (i.e., TP configuration) activate cortical regions as large as those activated in normalhearing guinea pigs by 1-octave-wide noise bands (Arenberg et al. 2000; Bierer and Middlebrooks 2002; present study). That result indicates that all of our electrical stimuli are likely to activate inhibitory sidebands of neuronal frequency response areas. Indeed, lateral inhibition probably shapes all the singlechannel cortical images that we record (Bierer and Middlebrooks 2002). Two-channel stimulation presumably further activates the lateral inhibitory surround areas. Nevertheless, lateral inhibition would suppress responses, not enhance them, as is demonstrated by reductions in thresholds. If lateral inhibition contributes to the channel interactions observed in the present study, it must be less prominent than other facilitatory factors.

The threshold shifts that we observed were most likely dominated by channel interaction that occurred within the cochlea. In the cochlea, we must consider direct vector summation of electrical stimuli as well as residual effects from charge stored on membranes and from activation of voltage-gated ion channels. Direct summation would have occurred in conditions in which there was no temporal offset between channels. Simple addition would predict that simultaneous in-phase stimulation of two nearby electrodes at equal levels would produce a current roughly $6 \mathrm{~dB}$ greater than the current from either electrode alone, although the exact value of the current increment would depend on details of local current paths, electrode impedances, and other factors. The expectation of current summation was borne out by the observed threshold shifts (e.g., Fig. $3)$. Threshold shifts were greater in the MP configuration than in the BP or the TP condition, presumably because greater cochlear current spread produced by the MP configuration resulted in greater overlap of current fields from the two electrodes. Current summation in the BP and TP configurations also is complicated by complexities of local field geometries resulting from multiple intracochlear active and return electrodes. Simultaneous stimulation with multiple BP and TP channels is worthy of further empirical and theoretical study.

The inverted-polarity condition represents a special case in which the observed results appear to reflect multiple complex geometries of current paths. We consistently observed an elevation of thresholds in the inverted-polarity simultaneous MP condition. In principle, one could model that condition as a vector subtraction of currents, although such a model would be complicated by nonhomogeneities in current paths. Intuitively, however, it is useful to regard this condition as reducing the net current flow from intracochlear source electrodes to excitable neural elements while increasing current flow from one intracochlear electrode to the other by way of a lowimpedance path through the perilymph. In effect, the two intracochlear electrodes in opposite polarity would function to some extent as a single bipolar pair, which we have shown to have a higher threshold than a monopolar electrode (Bierer and Middlebrooks 2002). All other inverted-polarity conditions tended to produce threshold reductions, although the likely mechanisms differ among conditions. In the condition of simultaneous BP stimulation of channels 2 and 5 , the return electrode of channel 2 (i.e., electrode 3 ) and the active electrode of channel 5 (electrode 5) were initially cathodal (and electrodes 2 and 6 were initially anodal), so one can think of that configuration as consisting of a double-sized active electrode (i.e., from electrodes 3 and 5) flanked by adjacent electrodes 2 and 6 . In the case of a temporal offset of $160 \mu \mathrm{s}$, the inverted-polarity condition effectively elongated the stimulus phase duration. That is, the cathodal second phase of the first biphasic pulse continued into the cathodal first phase of the trailing pulse. In our previous study (Bierer and Middlebrooks 2002), we found that cortical thresholds tended to be reduced by an average of $4.7 \mathrm{~dB}$ per doubling of phase duration, which is generally consistent with the present results from the $160-\mu$ s condition.

Channel interactions in same-polarity nonsimultaneous conditions (and inverted-polarity conditions with temporal offsets greater than $160 \mu$ s) must reflect a residual influence of the leading pulse on a trailing pulse. Residual effects could include stimulus current integrated by the resistance and capacitance of neural 
membranes and the activation of voltage-sensitive ion currents. Both of these factors presumably contribute to the time constant for summation of cochlear stimuli as measured with paired-pulse summation (e.g., Cartee et al. 2000) or with measures of chronaxie, which is the threshold duration of a pulse that is twice the amplitude of a threshold pulse of long duration (Loeb et al. 1983). Various investigators have reported such time constants in the range of roughly 150-500 $\mu$ s (Loeb et al. 1983; Cartee et al. 2000; van den Honert and Stypulkowski 1984; Dynes 1996). In the present study, for temporal offsets up to $\sim 640 \mu \mathrm{s}$, a subthreshold depolarization caused by a trailing pulse would have added to depolarization remaining from the leading pulse, thus reducing the threshold for activation by the trailing pulse. The longest temporal offsets used in the present study $(2000 \mu$ s) were longer than the range of reported time constants for cochlear electrical summation. We found that a subthreshold leading pulse tended to elevate slightly the threshold for a pulse that trailed by $2000 \mu$ s. One possible explanation for that observation is that the leading pulse would have had time to trigger activation of voltagesensitive potassium channels and inactivation of voltage-sensitive sodium channels, resulting in a partial refractory state and elevating the threshold for the trailing pulse (Hille 2001). Published time constants for refraction for scala tympani stimulation are around $1.5 \mathrm{~ms}$ (Dynes 1986; Javel et al. 1987; Parkins 1989; Bruce et al. 1999) and $0.7 \mathrm{~ms}$ for meatal stimulation (Cartee et al. 2000).

\section{Implications for speech-processor design}

Speech-processing strategies that are in use for cochlear implants can be divided into simultaneous analog and interleaved pulsatile strategies; recent designs also have presented pulsatile stimuli simultaneously to widely separated pairs of channels (Zimmerman-Phillips and Murad 1999). In simultaneous analog strategies, stimuli are presented to multiple stimulus channels simultaneously. Our results from simultaneous conditions show that channel interactions consistently were stronger in the MP configuration than in the $\mathrm{BP}$ or the TP configuration. For that reason, one would expect that speechprocessing strategies that use simultaneous stimulation would benefit from use of a BP or a TP configuration, which would result in reduced channel interaction and enhanced multichannel information transmission.

The rationale for use of interleaved pulsatile strategies is that nearby electrodes will never be stimulated simultaneously, thereby avoiding direct summation of current fields. Our results support that rationale by demonstrating that channel interactions, as reflected in threshold shifts, generally were substantially stronger in simultaneous compared with nonsimultaneous conditions. Our results show, however, that threshold shifts were not entirely eliminated by temporal offsets, but instead could persist for offsets of $640 \mu$ s or longer. Inasmuch as threshold shifts tended to decline with increasing temporal offsets, variation in the relative timing of pulses on any pair of channels could result in variations in the effective strength of the stimulus on each channel. The threshold shifts measured in nonsimultaneous conditions normally were only a few $\mathrm{dB}$ in magnitude, but a few $\mathrm{dB}$ is a substantial fraction of the dynamic range of cortical neurons in response to electrical cochlear stimulation.

The inverted-polarity condition that we tested has little practical relevance to any pulsatile strategy that presently is in use, since pulse trains normally would all be in the same polarity. That condition, however, is of some interest in relation to simultaneous analog strategies. In such strategies, the acoustic signal is filtered by a bank of bandpass filters, then the output of each filter is led to a cochlear electrode. Differences in the passbands of adjacent filters would result in differences in phase delays in the signals sent to each electrode, resulting in between-electrode phase differences of as much as $180^{\circ}$ for some frequencies. For that reason, the threshold elevations (in the simultaneous MP condition) or threshold reductions that we observed might mirror conditions that occasionally are present in clinical devices.

The present results likely underestimate the magnitude of channel interactions. Most of our analyses examined the effects of a subthreshold pulse on one channel on the responses to a pulse on a second channel. In practical use, speech processors present suprathreshold stimuli to multiple implant channels, so one would expect each channel to have substantial impact on the effectiveness of other channels. Also, we studied only single pulses on one or two channels, whereas any practical stimulus consists of modulated trains of pulses (i.e., in the case of a pulsatile strategy). Temporal integration among successive pulses on each channel presumably would have some influence on temporal details of interactions among channels. Despite these limitations, however, the results provide insights into the influence on channel interactions of temporal and spatial characteristics of pulses and lead to some considerations for speech-processor design.

\section{ACKNOWLEDGMENTS}

We thank Drs. Russell Snyder, Christopher Stecker, Ewan Macpherson, and Ian Harrington for their helpful comments on an early draft of the manuscript. Chris Ellinger 
provided essential engineering support. This work was supported by NIH grants RO1 DC04312, P30 DC05188, and NO1-DC-0-2108. Fellowship support (to JAB) was received from Hearing Research, Inc.

\section{REFERENCES}

Arenberg JG, Furukawa S, Middlebrooks JC. Auditory cortical images of tones and noise bands. J. Assoc. Res. Otolaryngol. 1:183194, 2000.

Battmer RD, Zilberman Y, Haake P, Lenarz T. Simultaneous Analog Stimulation (SAS) - Continuous Interleaved Sampler (CIS) pilot comparison study in Europe. Ann. Otol. Rhinol. Laryngol. Suppl. 177:69-73, 1999.

Bierer JA, Middlebrooks JC. Auditory cortical images of cochlearimplant stimuli: Dependence on electrode configuration. J. Neurophysiol. 87:478-492, 2002.

Bruce IC, Irlicht LS, White MW, O’Leary SJ, Dynes S, Javel E, Clark GM. A stochastic model of the electrically stimulated auditory nerve: Pulse-train response. IEEE Trans. Biomed. Eng. 46:630-637, 1999.

Cartee LA, van den Honert C, Finley CC, Miller RL. Evaluation of a model of the cochlear neural membrane. I. Physiological measurement of membrane characteristics in response to intrameatal electrical stimulation. Hear. Res. 146:143-152, 2000.

Cohen NL, Waltzman SB, Fisher SG. A prospective, randomized study of cochlear implants. N. Engl. J. Med. 328:233-237, 1993.

Drake KL, Wise KD, Farraye J, Anderson DJ, BeMent SL. Performance of planar multisite microprobes in recording extracellular single-unit intracortical activity. IEEE Trans. Biomed. Eng. BME-35:719-732, 1988.

Dynes SBC. Discharge characteristics of auditory nerve fibers for pulsatile electrical stimuli. Doctoral Dissertation, Massachusetts Institute of Technology, 1996.

Fishman KE, Shannon RV, Slatterly WH. Speech recognition as a function of the number of electrodes used in the SPEAK cochlear implant speech processor. J. Speech Lang. Hear. Res. 40:1201-1215, 1997.

Friesen LM, Shannon RV, Baskent D, Wang X. Speech recognition in noise as a function of the number of spectral channels: Comparison of acoustic hearing and cochlear implants. J. Acoust. Soc. Am. 10:1150-1163, 2001.

Furukawa S, Xu L, Middlebrooks JC. Coding of sound source location by ensembles of cortical neurons. J. Neurophysiol. 20:1216-1228, 2000.

Gantz BJ, Tyler RS, Knuston Jf, Woodworth G, Abbas P, McCabe J, Hinrichs J, Tye-Muarray N, Lansing C, Ku F, Brown C. Evaluation of five different cochlear implant designs: Audiologic assessment and predictors of performance. Laryngoscope 98:1100-1106, 1998.

Green DM, Swetts JA. Signal Detection Theory and Psychophysics. Wiley, New York, 1966.

Hille JB. Ion Channels of Excitable Membranes. Sinauer Associates, Inc., Sunderland, MA, 2001.

Hellman RP. Relation between the growth of loudness and highfrequency excitation. J. Acoust. Soc. Am. 96:2655-2663, 1994.

Hellweg FC, Kock R, Volrath M. Representation of the cochlea in the neocortex of guinea pigs. Exp. Brain Res. 29:467-474, 1977.

Javel E, Tong YC, Shepherd RK, Clark GM. Responses of cat auditory nerve fibers to biphasic electrical current pulses. Ann. Otol. Rhinol. Laryngol. 96(Suppl. 128):26-80, 1987.

Jolly CN, Spelman FA, Clopton BM. Quadrupolar stimulation for cochlear prostheses: modeling and experimental data. IEEE Trans. Biomed. Eng. 43:857-865, 1996.
Kral A, Hartmann R, Mortazavi D, Klinke R. Spatial resolution of cochlear implants: the electrical field and excitation of auditory afferents. Hear. Res. 121:11-28, 1998.

Loeb GE, White MW, Jenkins WM. Biophysical considerations in electrical stimulation of the auditory nervous system. Ann. NY Acad Sci. 405:123-136, 1983.

McDermott HJ, McKay CM. Pitch ranking with nonsimultaneous dual-electrode electrical stimulation of the cochlea. J. Acoust. Soc. Am. 96:155-162, 1994.

McKay CM, McDermott HJ. The perception of temporal patterns for electrical stimulation presented at one or two intracochlear sites. J. Acoust. Soc. Am. 100:1081-1092, 1996.

McKay CM, Remine MD, McDermott HJ. Loudness summation for pulsatile electrical stimulation of the cochlea: effects of rate, electrode separation, level, and mode of stimulation. J. Acoust. Soc. Am. 110:1514-1524, 2001.

Merzenich MM, White MW. Cochlear implant: The interface problem. In: Hambrecht FT, Reswick JB (Eds.) Functional Electrical Stimulation: Applications in Neural Prosthesis. Dekker, New York, pp 321-340, 1997.

Middlebrooks JC, Bierer JA. Auditory cortical images of cochlearimplant stimuli: Coding of stimulus channel and current level. J Neurophysiol. 87:493-507, 2002.

Najafi K, Wise KD, Mochizuki T. A high-yield IC-compatible multichannel recording array. IEEE Trans. Electron. Dev. 32:12061211, 1985.

Nuttall AL, Marques DM, Lawrence M. Effects of perilymphatic perfusion with neomycin on the cochlear microphonic potential in the guinea pig. Acta Otolaryngol. 83:393-400, 1977.

Osberger MJ, Fisher L. SAS-CIS preference study in postlingually deafened adults implanted with the CLARION cochlear implant. Ann. Otol. Rhinol. Laryngol. Suppl. 177:74-79, 1999.

PARkins CW. Temporal response patterns of auditory nerve fibers to electrical stimulation in deafened squirrel monkeys. Hear. Res. 41:137-168, 1989.

Plomp R. Aspects of Tone Sensation. Academic Press, London, 1976.

Ramachandran R, Davis K, May BJ. Single-unit responses in the inferior colliculus of decerebrate cats I. Classification based on frequency response maps. J. Neurophysiol. 82:152-163, 1999.

Redies H, Sieben U, Cruezfeldt OD. Functional subdivisions in the auditory cortex of the guinea pig. J. Comp. Neurol. 282:473488, 1989.

Shamma SA, Fleshman JW, Wiser PR, Versnel H. Organization of response areas in ferret primary auditory cortex. J. Neurophysiol. 69(2):367-383, 1993.

SHANNON RV. Multichannel electrical stimulation of the auditory nerve in man. I. Channel interaction. Hear. Res 12:1-16, 1983.

Skinner MW, Clark GM, Whitford LA, Seligman PM, Staller SJ, Shipp DB, Shallop JK, Everingham C, Menapace CM, Arndt PL, Antogenelli T, Brimacombe JA, Pijl S, Daniels P, George CR, McDermott HJ, Beiter AL. Evaluation of a new spectral peak coding strategy for the Nucleus 22 Channel Cochlear Implant System. Am. J. Otol. 15 (Suppl 2):15-27, 1994.

Spelman FA, Pfingst Be, Clopton BM, Jolly CN, Rodenhiser KL. Effects of electrical current configuration on potential fields in the electrically stimulated cochlea: field models and measurements. Ann. Otol. Rhinol. Laryngol. Suppl. 166:131-136, 1995.

Tong YC, Clark GM. Loudness summation, masking and temporal interaction for sensations produced by electric stimulation of two sites in the human cochlea. J. Acoust. Soc. Am. 79:19581966, 1986. 
Townshend B, Cotter N, Van Compernolle D, White RL. Pitch perception by cochlear implant subjects. J. Acoust. Soc. Am. 82:106-115, 1987.

van den Honert C, Stypulkowski PH. Physiological properties of the electrically stimulated auditory nerve. II. Single fiber recordings. Hear. Res. 14:225-243, 1984.

Wallace MN, Rutkowski RG, Palmer AR. Identification and localisation of auditory areas in guinea pig cortex. Exp. Brain Res. 132:445-456, 2000.

White MW, Merzenich MM, Gardi JN Multichannel cochlear implants: Channel interactions and processor design. Arch. Otolaryngol. 110:493-501, 1984.
Wilson BS, Finley CC, Lawson DT, Wolford RD, EdDington DK, RABINOWITZ WM. Better speech recognition with cochlear implants. Nature 352:236-238, 1991.

Young ED. Cochlear Nucleus. In: Sheperd GM (Eds.) The synaptic organization of the brain. Oxford University Press, New York.

ZENG FG, Turner CW. Binaural loudness matches in unilaterally impaired listeners. Q. J. Exp. Psychol. A 43:565-583, 1991.

Zimmerman-Phillips S, Murad C. Programming features of the CLARION multi-strategy cochlear implant Ann. Otol. Rhinol. Laryngol. Suppl. 177:17-21, 1999. 